Journal of Thermal Engineering, Vol. 6, No. 4, pp. 544-558, July, 2020

Yildiz Technical University Press, Istanbul, Turkey

\title{
EXPERIMENTAL STUDIES ON PRESSURE DROP CHARACTERIZATION OF CURVED TUBE SECTIONS IN LAMINAR FLOW REGIME
}

\author{
Vikram A. Kolhe ${ }^{1, *}$, Asmita D. Deshpande ${ }^{2}$, Ravindra L. Edlabadkar ${ }^{3}$
}

\begin{abstract}
In fluid metering technology, Coriolis Mass Flow Meter (CMFM) is the most prominent mass flow measuring instrument owing to its high accuracy, repeatability, and fidelity. The meter works on the principle of Coriolis force generated by the change in momentum of fluid, flowing through a vibrating tube. In CMFM, a straight or curved tube section is used to measure the mass flow rate of flowing fluid. The performance of such devices is found to vary with the shape and size of the curved tube and also with the flow regimes. Moreover, it has been reported to underpredict the flow rate than actual values in the laminar flow regime. In the recent past, few researchers had attempted to explain the phenomenon underlying the error in measurement with the help of secondary flow development in the laminar regime. The present investigations attempt to highlight the phenomenon with the help of pressure drops occurring in different sections of the tube. Characterization of hydraulic resistance of the system with respect to flow rate is thought to be a fundamental aspect to enlighten the issues. In this regard, an extensive experimental investigation has been undertaken on pressure drop characteristics in four tube shapes widely used in commercial CMFM such as U (Basic U and Narrow U), Omega, Delta and Diamond shape tubes in laminar flow regime. The results are expressed in terms of non-dimensional numbers for comparison.
\end{abstract}

Keywords: Pressure drop, Laminar flow regime, Friction factor.

\section{INTRODUCTION}

Flow measurement is an important task in many engineering applications. There are various methods available for flow measurement like differential pressure flow meters, displacement flow meters, and velocity flow meters etc., which indirectly measure the mass flow rate of a fluid. Whereas direct mass flow measurement devices like Coriolis Mass Flow Meter (CMFM) are, nowadays, gaining attention due to high accuracy and reliability. In practice, most of the times, flowing fluid is quite often turbulent and hence, the studies on flow field had been around turbulent flow regime. Generally, higher velocities have been found in the turbulent regime. However, as the fluid viscosity increases there can be increase in the mean velocity in the proportion such that the flow still remains laminar. The performance of CMFM is found to be deviated in this flow regime leading to underestimation of the flow rate as reported by Kumar and Anklin [1]. Bobovnik et al. [2] carried out numerical simulations to predict the performance of shell type Coriolis flow meter for a range of Re with the tube of two different aspect ratios. The velocity profile effect was presented in terms of anti-symmetric fluid forces which resulted in a loss of sensitivity at low flow rates. Kutin [3] presented a numerical study on the velocity profile effects for two straight tube configurations, one operating in a beam-type mode and other in a shell-type mode and reported that in both the cases, the meter sensitivity was affected. The author highlighted the intensive deviations in flow meter sensitivity at low Re. This can lead to a substantial economic loss in Petroleum industries. The literature review of CMFM in the laminar region has highlighted the development of secondary flow phenomenon in curved tube sections leading to under-read the meter in this region. The issue of under reading in case of CMFM needs attention and thereby remedial solution as an outcome of research.

This paper was recommended for publication in revised form by Regional Editor Jaap Hoffman Hoffman

${ }^{1}$ Amrutvahini College of Engineering, Department of Mechanical Engineering, Sangamner- 422608, Maharashtra, India.

${ }^{2}$ Shri Ramdeobaba College of Engineering and Management, Nagpur- 440013, Maharashtra, India.

${ }^{3}$ PVG's College of Engineering, Department of Mechanical Engineering, Nashik- 422004, Maharashtra, India.

E-mail address: ${ }^{1 *}$ kolhe.vikram@gmail.com, ${ }^{2}$ asmitadeshpande202@gmail.com, ${ }^{3}$ dredlabadkar@gmail.com

Orcid ld: ${ }^{1} 0000-0003-4395-1386,{ }^{2} 0000-0002-7469-3984,{ }^{3} 0000-0002-1699-3452$

Manuscript Received 22 September 2018, Accepted 18 December 2018 
The primitive work on the study of flow field was done in late 30's by Keulegan and Beij [4]. The fact of asymmetric velocity profile about the axis of pipe in a curved pipe due to change in flow direction has been revealed. It could be mainly attributed to the centrifugal force generating secondary currents across the cross-section of a pipe. This causes a shift in the location of the maximum velocity of flow from the centerline of pipe to an intermediate line between the center and the wall. The distortion of velocity profile leads to enhanced wall shear stress on the fluid and more pressure loss along the curved pipe compared to straight pipe. Total resistance coefficients calculated for bends with larger curvature radii as a function of the Re and the curvature ratio in the viscous and turbulent regimes were presented. The authors deduced critical Re for each bend selected. The investigations revealed the existence of three segments for a given curvature viz. the entrance to the bend which is termed as transition length, curved segment and downstream of the curved segment. It has been reported that the friction factor in the transition length increased from its value in the straight portion. The pressure drops between the point at the entrance to the bend and any point downstream of the bend were expressed in terms of effective friction factor. It was conjectured that effective friction factor could be a function of three prominent parameters viz. length to tube diameter ratio, curvature ratio, and Re.

Laminar flow in tubes requires larger length to attain its fully developed condition. The pressure loss in the developing region is always more as compared to the fully developed region. Thus characterization of pressure drop in the laminar regime for various CMFM tube configurations is expected to enlighten the phenomenon of secondary flow development in curved tubes causing pressure loss. It may lead to the selection of appropriate tube configuration for application of meter in laminar regime.

Limited theoretical and experimental investigations are carried out for the study of fluid flow in curved pipes in the laminar regime. Al-Nassri et al. [5] investigated laminar flow phenomenon in the entrance region of straight pipe with a circular cross-section. Experiments were conducted for entrance length of pipe with laminar and turbulent flow regime. The author derived the relationship between the viscous friction, the energy loss due to the lengthwise rate of change of kinetic energy coefficient and the total energy loss. The author compared the friction factor versus Re curve with standard curves for various entrance lengths and for a fully developed laminar region. They noticed a higher deviation in the curve at the entrance and after a Re of 1400 the deviation was small.

Cuming [6] solved the Navier-Stokes equation for the flow through curved pipes with first and second power series of curvature of the pipe. The author presented the effect of aspect ratio of pipe on the intensity of secondary flow and reported that for aspect ratio near unity, the velocity increases in the outer half of the bend and decreases in the inner. He also compared the curved pipes of circular and square sections and found that the secondary flow in a square section was stronger than that in a circular section. Fargie and Martin [7] presented a theory for prediction of flow development and pressure drop at the inlet of circular pipe in laminar regime. They developed simpler expression using both differential and integral momentum equations. However, it has been reported that the center line velocity gets over predicted values in the intermediate stages along the developing length. Average values of friction factors and pressure drops were claimed to predict the experimental data correctly.

In commercially developed CMFM, straight or curved tube sections (with various configurations) are employed to measure the mass flow rate. Gupta P. et al. [8] conducted experimentation with four tube configurations to identify sensor positions for the maximum phase shift between two limbs of the tube. Sharma et al. [9] investigated the performance of Coriolis flow meter for three different sets of $U$ tube configurations with the effect of parameters such as sensor position and drive frequency for minimum percentage error in measurements. Ahn and Uslu [10] experimentally studied the pressure drop characteristics of straight and corrugated helically coiled configurations of $10 \mathrm{~m}$ length in fully turbulent flow and noticed an increasing trend of friction factor with an increase in pipe diameter.

From the literature survey, it was found that the secondary flow analysis and pressure drop characterization of curved tubes having a fixed geometrical shape in the laminar regime had not been reported. Hence, towards the understanding of the development of secondary flow phenomenon in CMFM tube leading to underpredict the meter performance, it is necessary to study the flow development and pressure drop characteristics in laminar regime in a steady tube of different configurations. 
Journal of Thermal Engineering, Technical Note, Vol. 6, No. 4, pp. 544-558, July, 2020

\section{SELECTION OF TEST SECTION CONFIGURATIONS}

Literature review reveals limited information regarding the response of tube geometries to pressure drops over a range of $\operatorname{Re}<2300$. In pursuit of issues of erroneous readings by CMFM in laminar flow, characterization study would be pivotal in exploring flow physics. Based on information of commercially available CMFM, four basic tube shapes are selected in the present investigations; those are U (Basic U and Narrow U), Omega, Delta, and Diamond shape. The past studies, as well as commercial catalogues, do not provide directives for dimensions regarding tube shapes. So, the dimensions of tubes for each configuration have to be worked out considering constraints such as space for test sections and tube material. One of the objectives of the present investigation program put forth is the study of flow pattern transition through flow visualization under steady state condition. This demands transparency of test section for which materials like glass or acrylic are best-suited candidates. However, molding of such materials to get desired configurations maintaining reasonable accuracy in dimensions has been found quite challenging by manufacturers. The steady state nature of the flow field in a non-vibrating condition does not get affected by the modulus of elasticity of tube material which is totally different than the metals used in CMFM. This has been also identified as a cost-intensive job. Conventional transparent rubber tubes when examined can show too much flexibility to maintain configuration as well as relatively more roughness offering frictional resistance. Taking cognizance of all these constraints, transparent Polyurethane (PU) tube is selected which renders adequate stiffness so as to act as a rigid conduit. A prime advantage offered by PU tubes would be its hydrodynamic smoothness compared to metallic tubes. This could make one to focus on pressure drop due to configurations of test sections rather than friction offered by the tube material. As per market availability, a circular tube of inner diameter $12 \mathrm{~mm}$ and outer diameter $16 \mathrm{~mm}$ is taken to form above mentioned shapes. Pressure tapings are made at appropriate locations along the length of the tube and blue color PU tubes with outer diameter $6 \mathrm{~mm}$ are used for connecting these tapings with the common manifold as shown in figure 3(b). Since the entire investigation program is covering the laminar flow regime, the entrance length for test sections has been maintained the same. The empirical relation for the entrance length [11] is given by the following equation,

$$
\mathrm{L}_{\mathrm{H}}=0.06 \times \operatorname{Re} \times \mathrm{d}
$$

It yields maximum length of $1500 \mathrm{~mm}$ ensuring fully developed flow entering each tube section. For all these shapes the length of the tube forming a particular shape is kept fixed to approximately $1000 \mathrm{~mm}$ for comparative study. The geometries of selected tube shapes have been chalked out considering the feasibility of PU material to take the shapes in a fixed length of $1000 \mathrm{~mm}$. The inlet and outlet bends could be made as sharp as to get inner radius as $50 \mathrm{~mm}$. This was maintained in all tube sections. Selected configurations for tube sections are categorized into two broad groups; A) Tube sections with gradual curves B) Tube sections with the sharp curves.

As seen in figure 1(a), Basic $U$ shape is formed taking the radius of curvature of $112.5 \mathrm{~mm}$ which makes the distance between outer sides of the limbs equal to $225 \mathrm{~mm}$. The inlet and outlet limbs are considered three times of the curvature radius. Whereas in Narrow $U$ shape tube, curvature radius is reduced by $37.5 \mathrm{~mm}$ through a proportionate increase in limbs preserving the total length of the test section as depicted in figure 1(b). Another configuration for tube section with gradual curve taken is Omega-shaped. It is basically circular configuration with a suitable opening by removal of a sector of the circle as shown in figure 1(c). The inlet and outlet are connected to the straight tube sections as practiced in previous cases through bends. 
Journal of Thermal Engineering, Technical Note, Vol. 6, No. 4, pp. 544-558, July, 2020

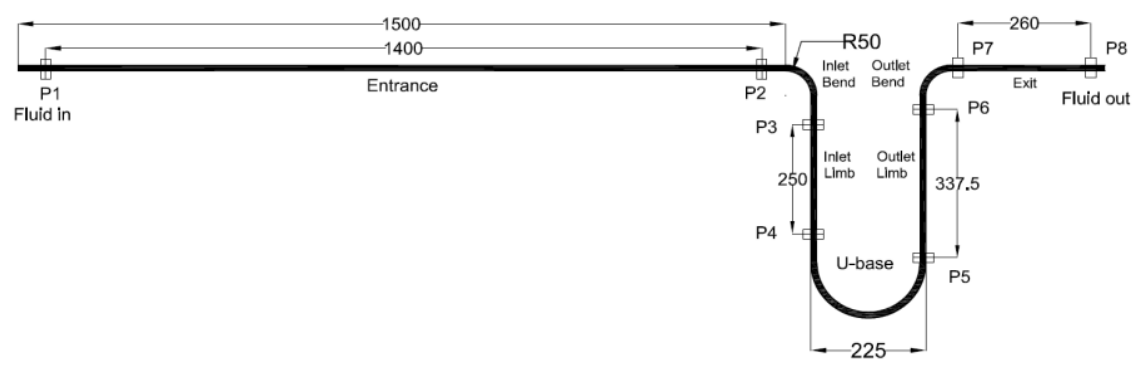

a. Segments of Basic U shape tube

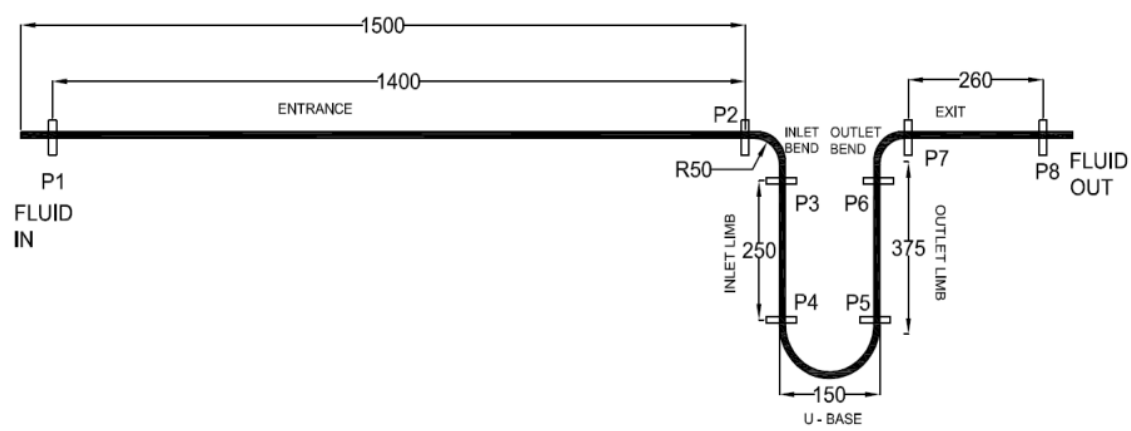

b. Segments of Narrow U shape tube

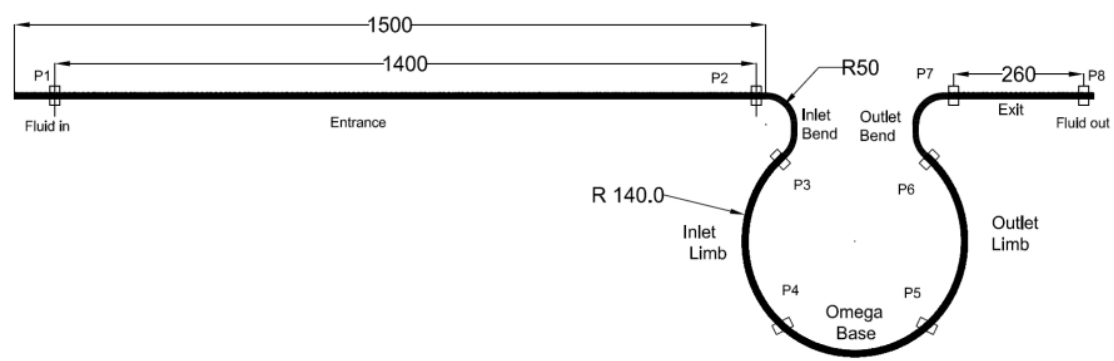

c. Segments of Omega shape tube

Figure 1. Tube sections with gradual curves

The second category of tube configurations comprises of tube sections with sharp bends. As mentioned earlier two such configurations viz. Delta and Diamond shapes are picked up for investigations. Delta shape is made of two sharp bends subtending an angle of $60^{\circ}$ at the base. Thus, remaining two inclined limbs together form an equilateral triangle as seen in figure 2 (a). The final tube section takes shape of a section cut at the top of an equilateral triangle. When one more bend gets added in the previous configuration another tube section configured in a Diamond shape can be formed. As shown in figure 2(b), to conform the restrictions of tube length in a configuration (i.e. $1000 \mathrm{~mm}$ ) the Diamond is formed to take a shape of rhombus cut at its one corner for inlet and outlet connections. 


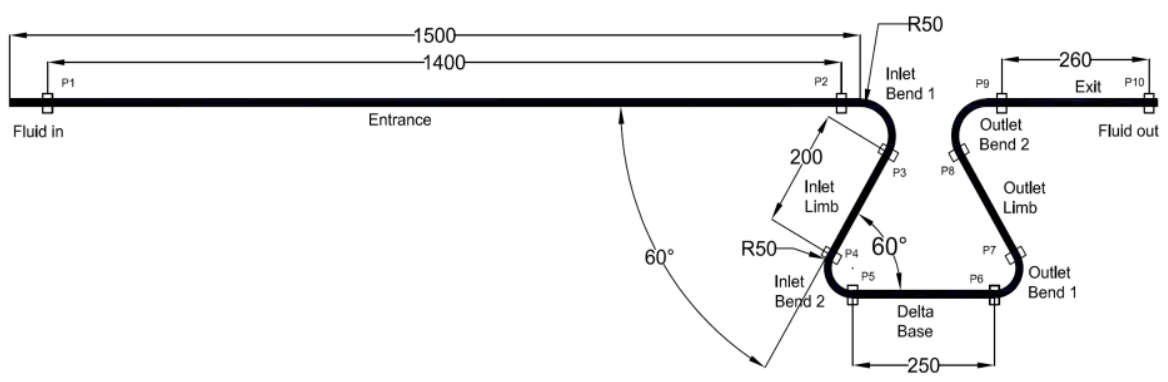

a. Segments of Delta shape tube

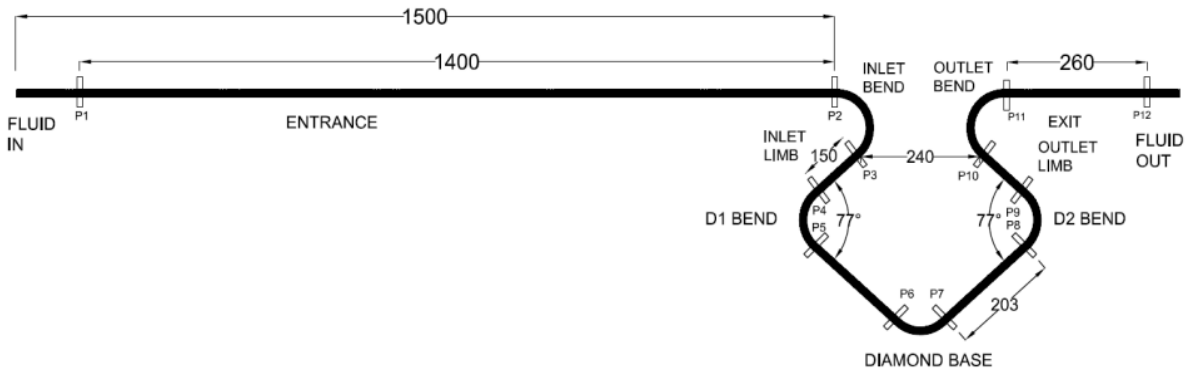

b. Segments of Diamond shape tube

Figure 2. Tube sections with sharp curves

\section{DEVELOPMENT OF TEST FACILITY}

To maintain steady flow rates in the laminar region, a constant flow water supply from a reservoir having a capacity of 45 liters and elevation of $1 \mathrm{~m}$ has been provided. Elimination of the pump results in steady flow rates and ease of control of fluid flow. The desired flow rate is obtained by proper positioning of a control valve and maintaining the liquid level in the supply tank. From elementary calculations, it could be realized that overall pressure drop expected to remain below 10,000 Pa. Hence, the supply tank has been elevated to $1 \mathrm{~m}$ height above the test section as shown in figure 3 (a). For the sake of accurate and precise measurement of pressure drop in different sections, use of $\mathrm{U}$ tube differential manometer has been envisaged. Since the study is limited to the laminar regime of fluid flow Carbon Tetrachloride $\left(\mathrm{CCl}_{4}\right)$ is found as a viable option. It is an organic substance occurring in a liquid state at ambient conditions and immiscible with water. Being a colorless liquid, iodine needs to be added in a small amount as a marker to turn it into violet color for visibility of meniscus.

To measure the pressure drop in various segments of the tube, each test section has been fragmented into appropriate segments for characterization of pressure drop. Figure 3 (b) represents the provision of pressure taps in Basic U shape tube section. Pressure taps are made with plastic tubes of $4 \mathrm{~mm}$ ID having sufficient stiffness and then connected by PU tubes to the manifold. Specially designed stainless steel manifolds were fabricated to serve as common headers at two limbs of the manometer for pressure measurement. Ball valves made in brass with suitable pipe size diameter are provided at the inlet and outlet of the test section to control the flow rate. The water properties such as density and viscosity are considered at the temperature being its strong function than pressure. Water temperatures are noted with the ordinary thermometer. The flow is controlled through outlet ball valve opening to set at a $\mathrm{Re}$ in a specified range. This ensures the entire tube always filled with water leaving no room for the air bubble. The flow visualization has been treated as one of the tools supporting the arguments behind pressure drop measurements and thereby response in friction factor. Hence, a technique of die injection has been envisaged using Potassium Permanganate $\left(\mathrm{KMnO}_{4}\right)$. Only representative photographs of flow pattern transition for Basic U shape and for bends in Diamond shape has been put up in the discussion. 
Journal of Thermal Engineering, Technical Note, Vol. 6, No. 4, pp. 544-558, July, 2020

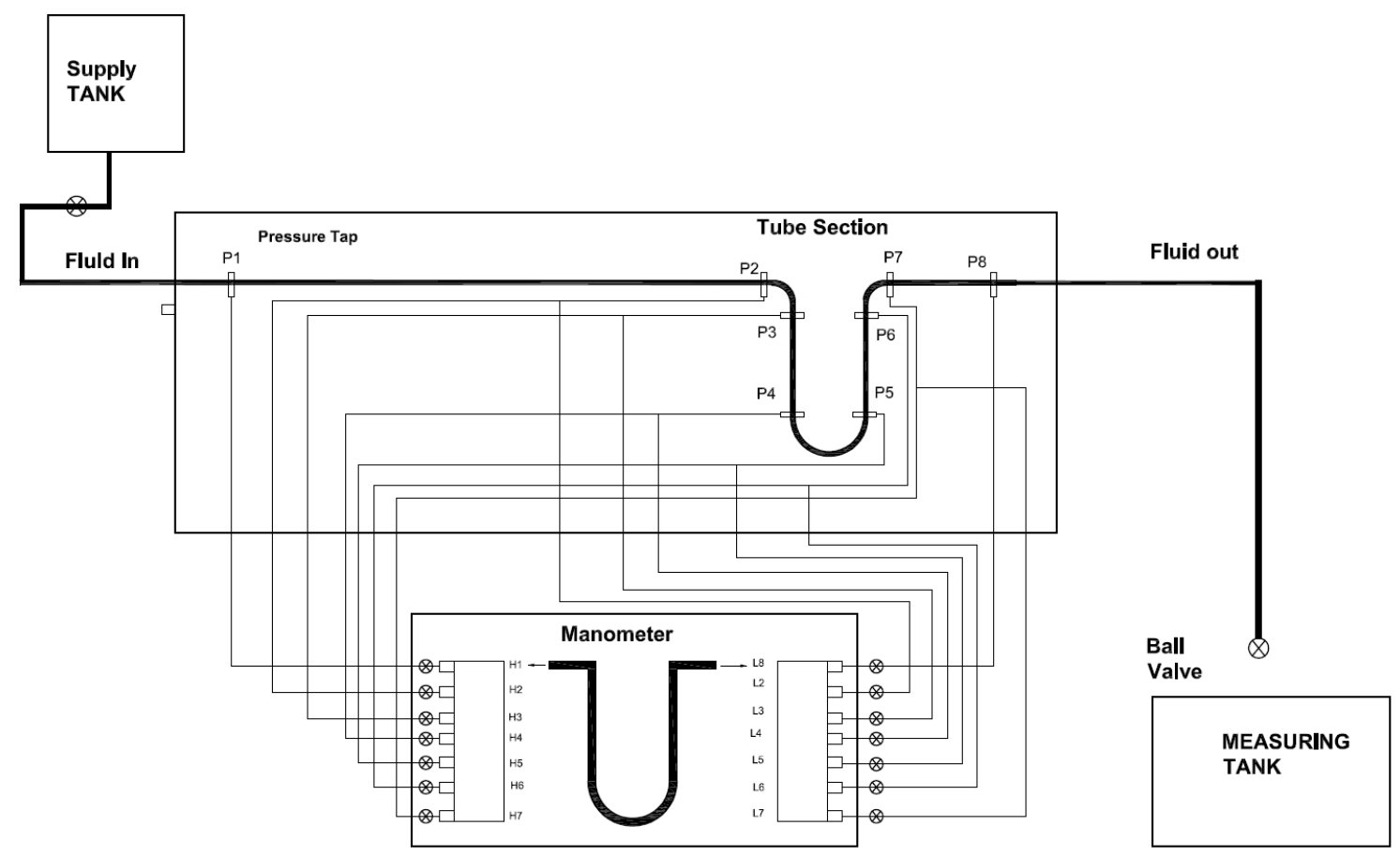

a. Schematic diagram of experimental setup

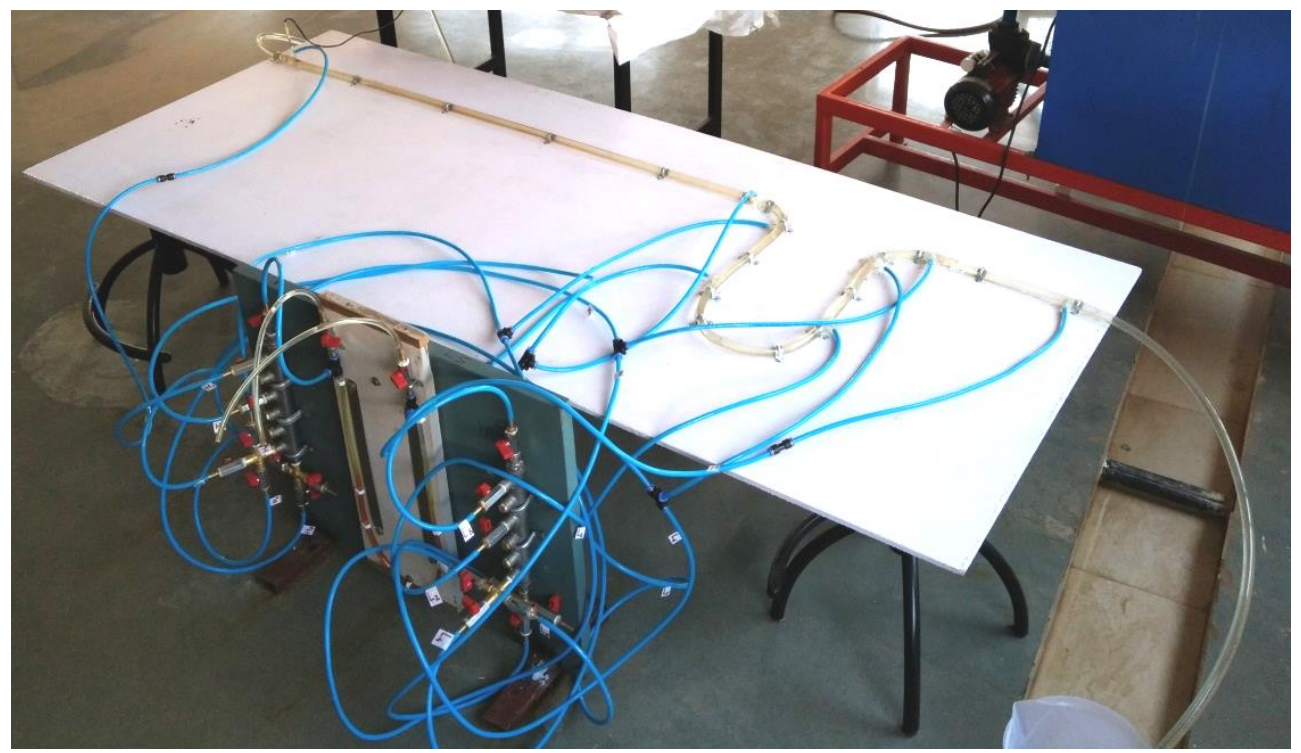

b. U shape test section with pressure taps

Figure 3. Experimental set up

The uncertainties existing in measurement during experiments are found to be $5 \%$ in volume measurement, below $1 \%$ in time count and $6.9 \%$ in level difference measurement in manometric level difference. During experimentation the temperature of manometric fluid (i.e. $\mathrm{CCl}_{4}$ ) as well as working fluid (i.e. water) is found to be constant within $\pm 0.5^{\circ} \mathrm{C}$. Hence, the densities of these fluids can be considered constants for all practical purposes. 


\section{RESULTS AND DISCUSSION}

One of the objectives of the present investigation is to work out friction factor response in a curved tube section over that of a straight tube of the same length. Darcy friction factor $\left(f_{c}\right)$ in the curved segment of a tube has been worked out from the measured pressure drop as given by equation (2),

$$
\mathrm{f}_{\mathrm{c}}=\frac{\Delta \mathrm{P}}{\left(\frac{1}{2} \rho \mathrm{U}^{2}\right)\left(\frac{1}{\mathrm{~d}}\right)}
$$

At the entrance point of each tube section, it has been ensured to have fully developed flow through the provision of maximum required entrance length. The flow front as enters through inlet bend into the given test section has to again succumb developing zone through successive curved paths. Hence, the pressure drop in the tube of the same length as that of tube section cannot be calculated from a simple correlation for friction factor. In this regard, the analytical approach developed by Al-Nassri et.al. [5] has been followed for calculating the friction factor in developing length. It has been proposed by the authors to consider the composition of viscous friction $\left(\mathrm{f}_{\mathrm{v}}\right)$ and the change in kinetic energy $\left(f_{k}\right)$ to calculate friction factor $\left(f_{s}\right)$ in a straight tube.

It has been an established fact that fully developed flow has parabolic nature of velocity profile in laminar regime. So the wall shear stress $(\tau)$ varies linearly to the velocity gradient in a linear direction. However, it can be argued that the wall shear stress would have a relationship with velocity gradient through a power law as,

$$
\tau=\mu_{\text {apparent }}\left(\frac{\mathrm{dU}}{\mathrm{dr}}\right)^{\mathrm{n}}
$$

Thus, the fluid moving in the developing region has been treated at par with a non-Newtonian fluid [5]. Index ' $n$ ' in equation (3) has a scope from zero to one and it is influenced by the radius of the tube $\left(r_{o}\right)$, axial distance (X) and Reynolds number (Re). The two parameters relating to the length can be non-dimensionalized in terms of (Z).

$$
\mathrm{Z}=\frac{\mathrm{X}}{\mathrm{r}_{\mathrm{o}}} \text { and } \xi=\frac{\mathrm{Z}}{\mathrm{R}_{\mathrm{e}}}
$$

The theoretical relationship between ' $\mathrm{n}$ ' and these parameters in non-dimensional form has been given as [5],

$$
\mathrm{n}=214.28(\xi)^{3}-102.52(\xi)^{2}+17.05(\xi)
$$

The authors [5] proposed the component of friction factor due to change in kinetic energy $\left(\mathrm{f}_{\mathrm{k}}\right)$ as given below,

$$
f_{k}=\left[\frac{2\left(117 n^{2}+102 n+21\right)}{\left(10 n^{2}+11 n+3\right)^{2}}\right]\left[\frac{\partial_{n}}{\partial_{z}}\right]_{R_{e}}
$$

Where, the first bracketed term stands for the correction factor to account for kinetic energy. To address to the second bracketed term in equation (6), the differentiation of equation (5) over ' $Z$ ' when carried out for the fixed value of Re yields equation (7),

$$
\left[\frac{\partial_{\mathrm{n}}}{\partial_{\mathrm{z}}}\right]_{\mathrm{R}_{\mathrm{e}}}=\left[\frac{642.8}{\mathrm{Re}^{3}}\right] \mathrm{Z}^{2}-\left[\frac{205}{\mathrm{Re}^{2}}\right] \mathrm{Z}+\frac{17.05}{\mathrm{Re}}
$$

In this way for a given set of Re, equation (6) is employed to finally work out value of $\left(\mathrm{f}_{\mathrm{k}}\right)$. The viscous dissipation component (fv) of total friction factor is computed by the expression [5],

$$
\mathrm{f}_{\mathrm{v}}=8\left(\frac{\mu}{\rho \mathrm{Ud}}\right)\left(\frac{\mathrm{D}}{\mathrm{U}}\right)^{1-\mathrm{n}}\left(\frac{6 \mathrm{n}+2}{\mathrm{n}}\right)^{\mathrm{n}}
$$


The total friction factor in developing flow in a straight tube is then expressed by equation (9),

$$
\mathrm{f}_{\mathrm{s}}=\mathrm{f}_{\mathrm{v}}+\mathrm{f}_{\mathrm{k}}
$$

From the ratio of equation (2) to equation (9) the response of frictional resistance due to curvature effect over its counterpart straight tube sections of same lengths has been computed over the set of Re as shown in figure 4 .

It can be seen from figure 4, the ratio of friction factor is responding to the Re in similar trends for all the configurations. Basic $\mathrm{U}$ shape tube encounters the least resistance to fluid flow as compared to other. There are two noteworthy points in this plot viz. the scatter over the flow range and the resistance offered by tube sections. When tubes sections having $U$ shape are considered, the resistance offered to the flow in Basic U shape is found to be minimum. With narrowing down the radius of curvature of $U$ section while keeping the effective length same has indicated a quite similar trend. The apparent parallelism between these two trend lines simply delineates the fact that the difference is nearly with reference to an increased value of friction factor $\left(f_{c} / f_{s}\right)$ for the same Re. With the sharper curvature in $\mathrm{U}$ shape more is the scatter. This could be attributed to the departure of flow pattern from laminar to transition region as visualized in figure 6. It shows that although flow is globally treated in the laminar region, the local curvature effects induces disturbance to the flow causing it to digress from a streamlined pattern. Omega shape tube section which is another member of gradual curvature reveals the steepest decline in friction factor ratio compared to its counterparts under study. The reason may be the continuous curved path to the flow unlike in other tube sections.

The study of characterization of tube sections with sharp bends exhibits more pressure drop over a flow range of Re when compared to those with gradual curves. The straight segments occurring intermittently between successive bends contribute to the development of flow. However, as mentioned earlier the developing length is found insufficient. The sharp corners in these tube sections additionally contribute the drop in pressure. The plot also indicates enhanced friction offered by Diamond shape tube than Delta shape as anticipated by the presence of extra bend than in latter. Tube length for both sections being same considering practical constraints, the angle of bend doesn't influence friction factor for small values of Re. However, the presence of additional bend is more pronounced as Re approaches to its limit of the laminar regime.

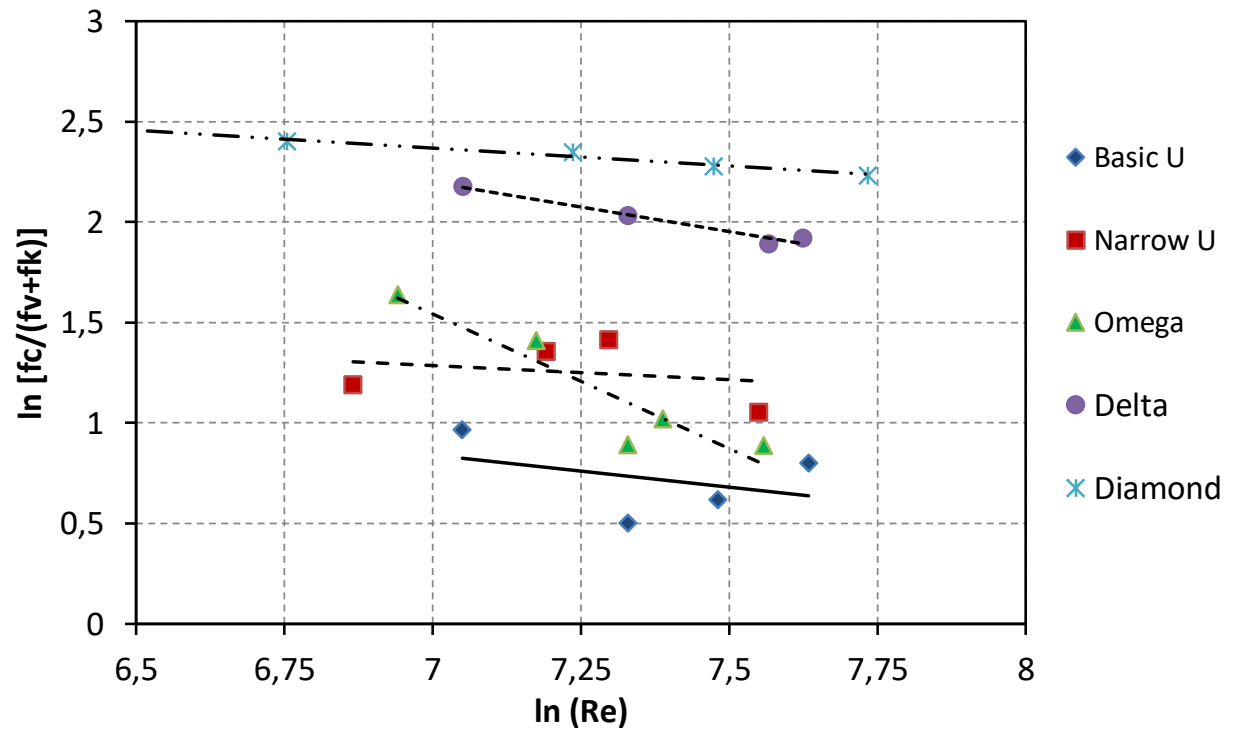

Figure 4. The response of $f_{c} /\left(f_{v}+f_{k}\right)$ in laminar flow regime for various tube configurations

With the help of a test facility developed as shown in figure 3 (a), experimental pressure drop characterization has been carried out for all the test sections. Each tube section is fragmented into a number of segments covering its characteristic features like bends or curved paths, straight portions etc. Thereby, the 
characterization of each tube section has been accomplished through the measurement of pressure drops in various segments. Segment wise pressure drop measurements enable to arrive at some rationale for providing an explanation of the phenomenon shown in figure 5. Basic U shape, when taken for investigation, is fragmented for pressure tappings into five segments starting from the inlet point to the exit of the entire tube section. Pressure tappings are taken such that the characteristic geometries are covered as indicated in figure 1 (a). Pressure drops in segments like inlet bend to $U$ base indicate similar trends except for the change in their numerical values.

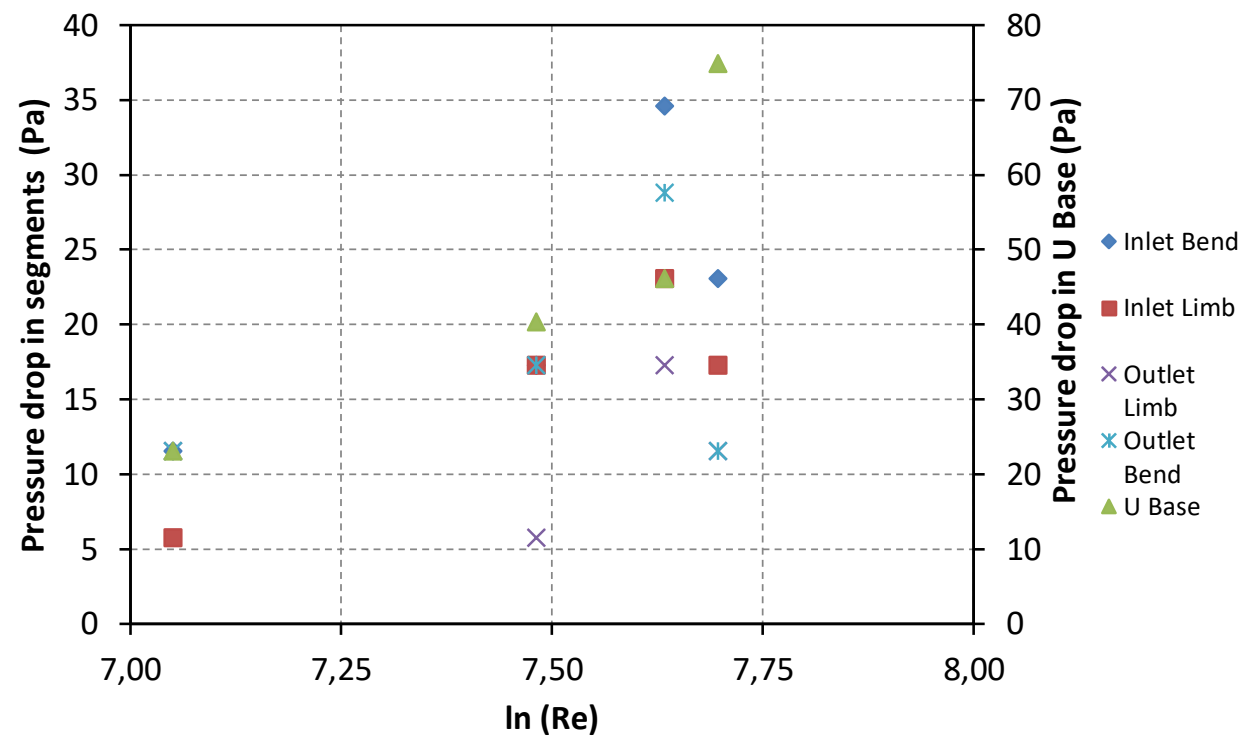

Figure 5. Pressure drop in different segments of $U$ shape tube section

The bends at inlet and outlet of the tube sections are $1 / 6^{\text {th }}$ of the length of straight limbs exhibit lower pressure drops. The flow in U base segment has produced more pressure drop than in rest of the segments. This could be attributed to the continuous curvature, the segment length being 1.3 times larger than inlet and outlet limbs. Compared to inlet limb, outlet limb has shown to offer somewhat less resistance in spite of the similar distance between pressure taps. This could be supported by the existence of smooth curvature in the preceding segment of the outlet limb. Visualization of transition of flow pattern in a $U$ curve (the portion of tube between inlet and outlet limbs) can be seen in figure 6 in support of the arguments stated earlier. The gradual flow development between two pressure taps has added to enhanced friction factor. The dispersion of injected die has been observed more as the Re approaches its critical value of 2300. This clarifies enhanced turbulence effect adding turbulent viscosity in the pattern.

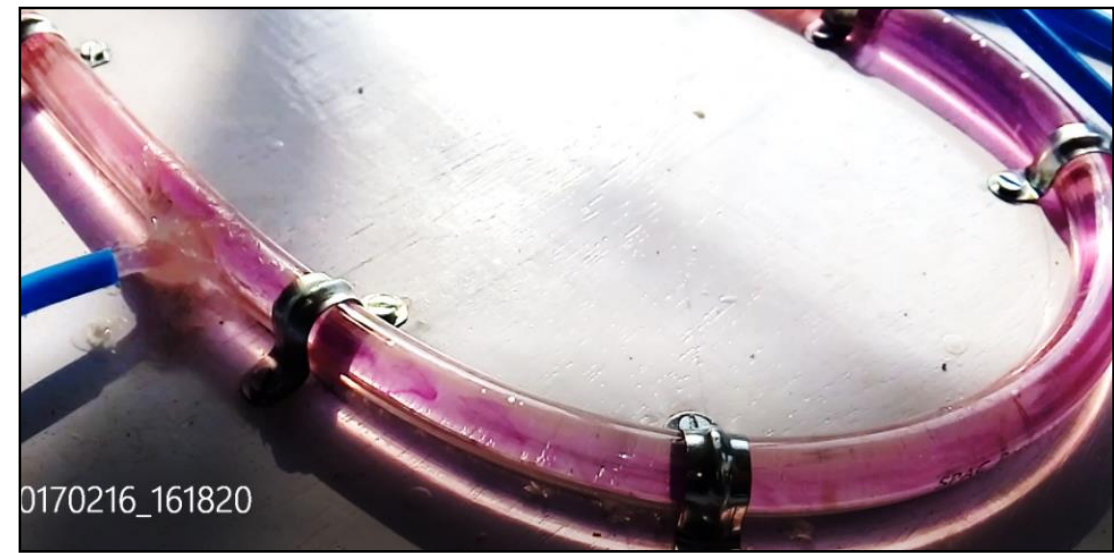

Figure 6. Flow visualization in a $U$ curve (the portion of the tube between inlet and outlet limbs) of $U$ shape tube section 
Further, the curvature effect of Basic $\mathrm{U}$ shape has been investigated. The diameter of Basic $\mathrm{U}$ shape gets narrowed down to around $2 / 3$ of its original diameter restrained by the flexibility of PU tube. It has to be noted that in spite of the reduction in diameter the distance separating two pressure tapings is maintained exactly same as that in the previous case. The pressure drops obtained in similar fragments differ considerably as exhibited in figure 7. The rising trends in pressure drop with the rise in Re can be experienced very much similar as in case of Basic U shape. However, the pressure drops in inlet and outlet limbs are distinct in this case with outlet limb revealing enhanced pressure drop than inlet limb. This could arise from spontaneous mixing of fluid particles leaving the sharp curvature. Another point of distinction between Narrow U shape and in its precursor case is pressure drop observed in bends. Unlike the previous case, the outlet bend has pronounced drop in pressure than its inlet by 4 to 5 times. Also, the scatter in the measured values can be seen. The reduced pressure drop observed in Narrow U shape than in Basic U shape can be solely explained with increased straight limbs between two pressure tappings. These equally increased straight limbs at the leading and trailing sides of the narrowed $U$ curvature provide an adequate developing zone for flow.

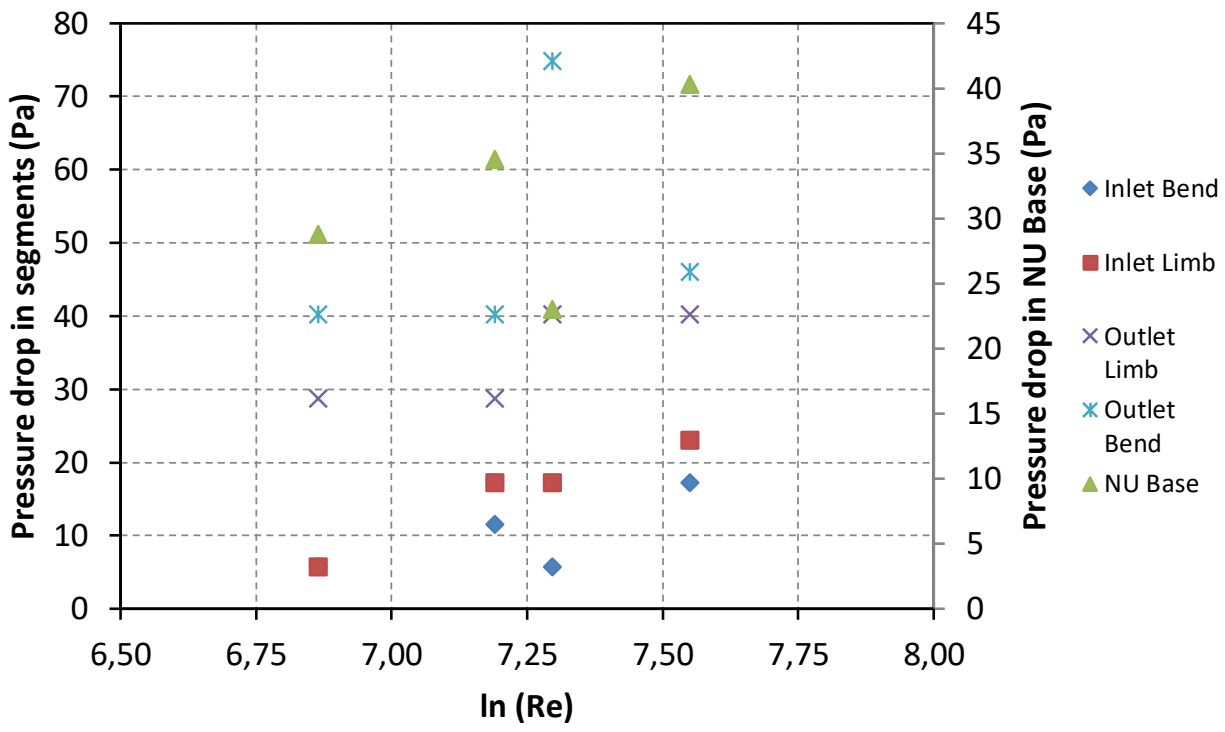

Figure 7. Pressure drop in different segments of Narrow U shape tube section

In case of a gradually curved tube with Omega shape, the test section is fragmented into the number of segments as in the previous case. Although the radii of bends at inlet and outlet are chosen the same as in U shape tubes, the curved paths are increased rendering obtuse angle between two pressure taps. This has contributed to the highest pressure drops over the entire range of Re than in rest of the cases as shown in figure 8 . The angle between pressure taps at Omega base being less than $180^{\circ}$ reveals little fall in pressure drop than its counterpart studied in the previous cases. Unlike the $U$ shapes the limbs at upstream and downstream sides of Omega base are curved. Thus almost for the similar distance between pressure taps, the pressure drops can be seen little higher as expected. The continuous curvature in this tube section with the absence of any straight path can support the steepness of friction factor curve as indicated in figure 4. The scattered measurement is another characteristic observation on record as seen in figure 8 . The symmetry between limbs resulted in the close cluster of data points. 


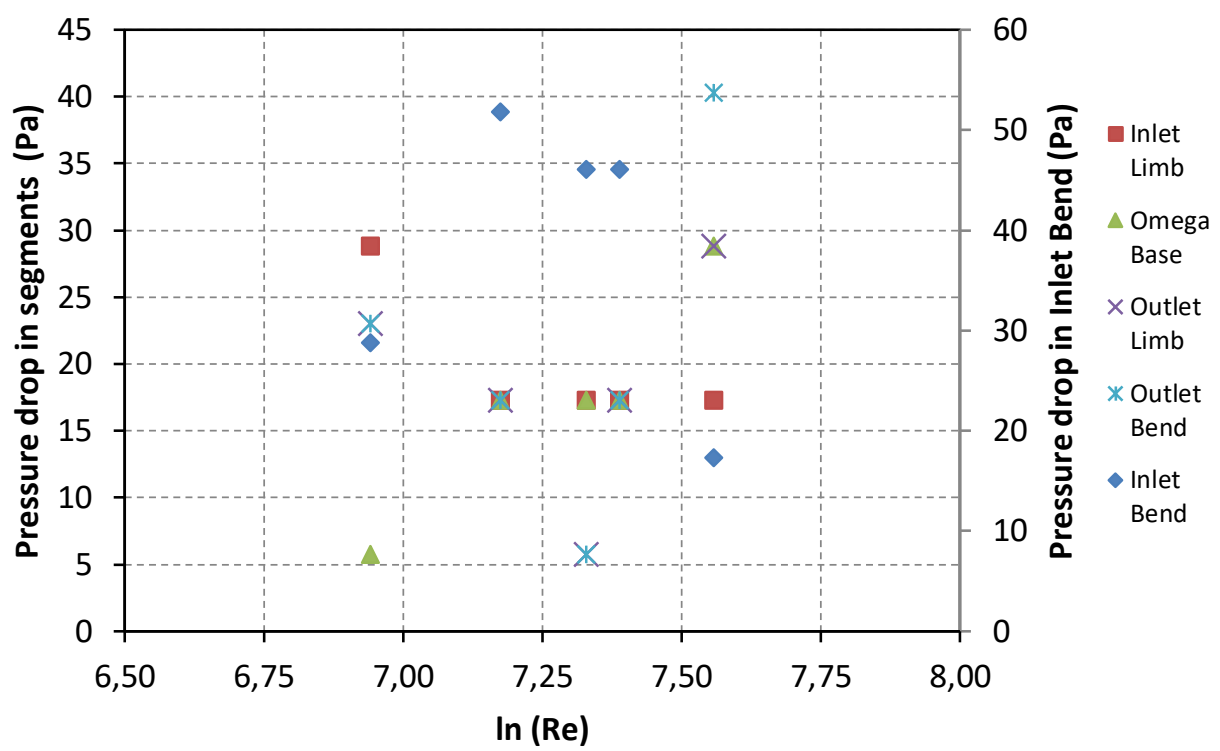

Figure 8. Pressure drop in different segments of Omega shape tube section

The response of friction factor $\left[\mathrm{f}_{\mathrm{c}} /\left(\mathrm{f}_{\mathrm{v}}+\mathrm{f}_{\mathrm{k}}\right)\right]$ to Re when fluid flows through sharply cornered tube section can be also understood through observations of pressure drops in sub-sections. The first in this category is Delta shaped tube section having four sharp bends. The base angles subtended by inlet and outlet limbs are maintained to $60^{\circ}$ as stated earlier forms an equilateral triangle. The configuration is exactly symmetric about the vertical axis which is also a perpendicular bisector of the base. Figure 4 points out the fact that the enhanced friction factor in Delta shape by an order of 2 to 2.5 times as that in Basic $U$ shape tube. Amongst the participating limbs in this shape, outlet limb has shown higher pressure drop as indicated in figure 9. This could be explained due to increased local turbulence owing to a continuous disturbance in its preceding limbs. Pressure drop in each segment is higher as compared to previous cases. Increase in Re lead to higher incremental pressure drop in Delta base, outlet limb and outlet bend 2.

Similar to Delta tube section, Diamond shaped tube section has also exhibited the highest pressure drop which is anticipated due to the addition of the fifth bend in Delta section. However, the friction factor is sloping down with the rise in Re at a little slower rate than in case of Delta section as shown in figure 4 . The overview of pressure drops in various segments is depicted in figure 10.

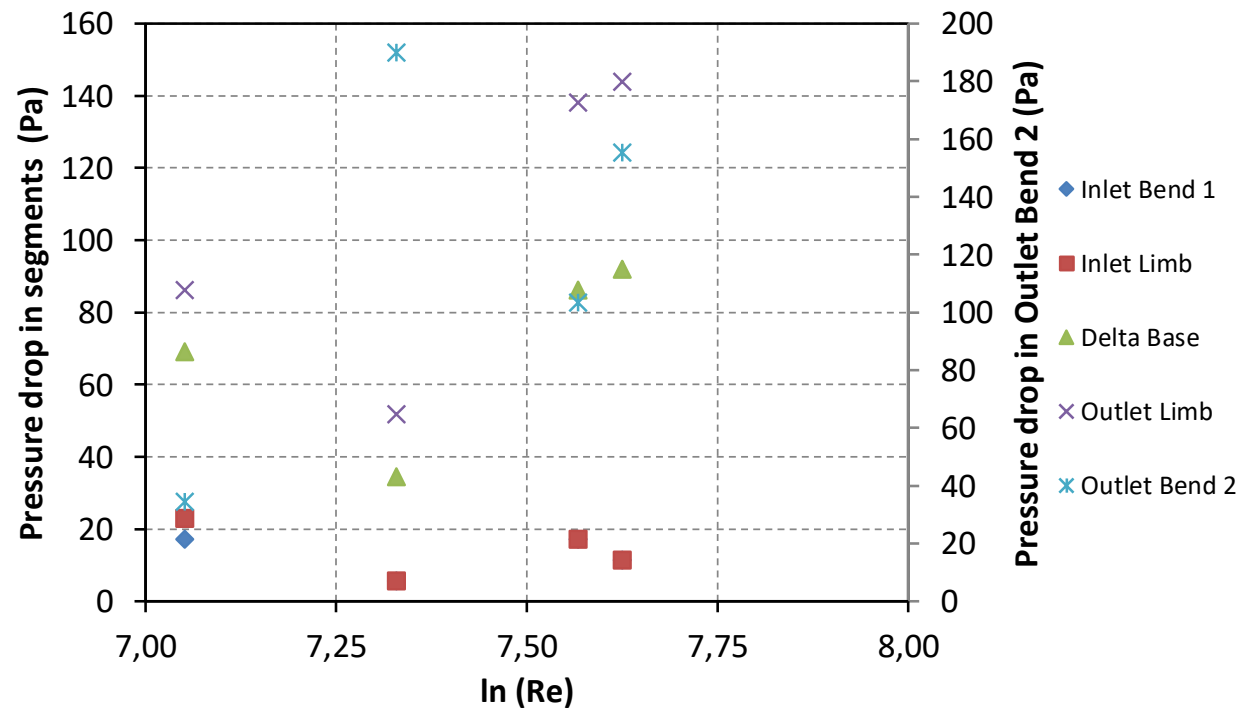

Figure 9. Pressure drop in different segments of Delta shape tube section 
The diamond shape is basically in the form of a kite which is a special case of a quadrilateral. The inlet bend like in Delta shape is obtuse, needs longer developing length once the fluid has passed through it. But the available length in the inlet limb being smaller as seen in figure 2(b), the pressure drop is more than observed in the rest of the limbs. The complexity of the Diamond base has made the overall pressure drop in the tube section to remain at the highest amongst all tube sections. The inability of flow to dissipate its energy through turbulence when the flow is low, especially for Re less than 1300 has shown more fluctuations in measurements. Even this could be seen from the deviation of data points from the main trends.

The highest values of pressure drop observed in this tube section can be well understood from figure 11 which clearly indicates disturbed flow in a diamond base (the portion of the tube between D1 and D2 bend) of the tube section. The injected die shows wavy nature more near to tube wall due to the sharp bend indicating turbulent viscosity. Similar observations were noted in all three bends.

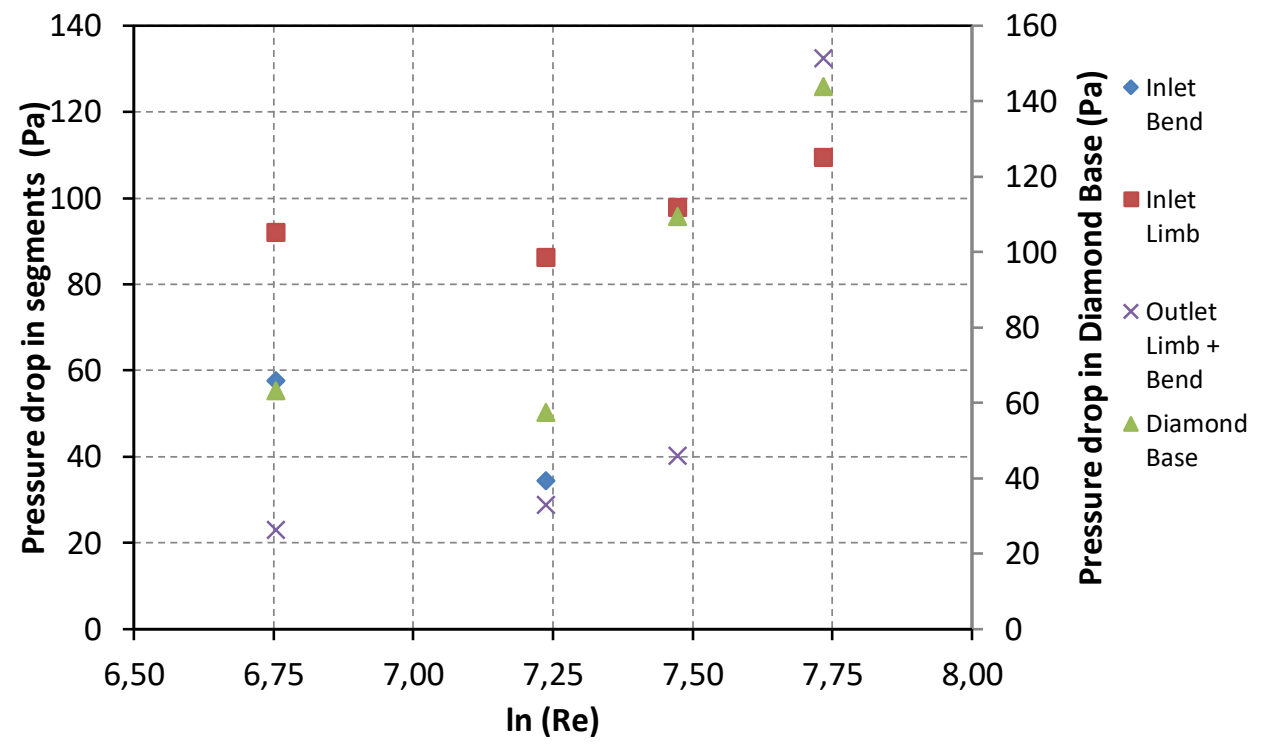

Figure 10. Pressure drop in different segments of Diamond shape tube section

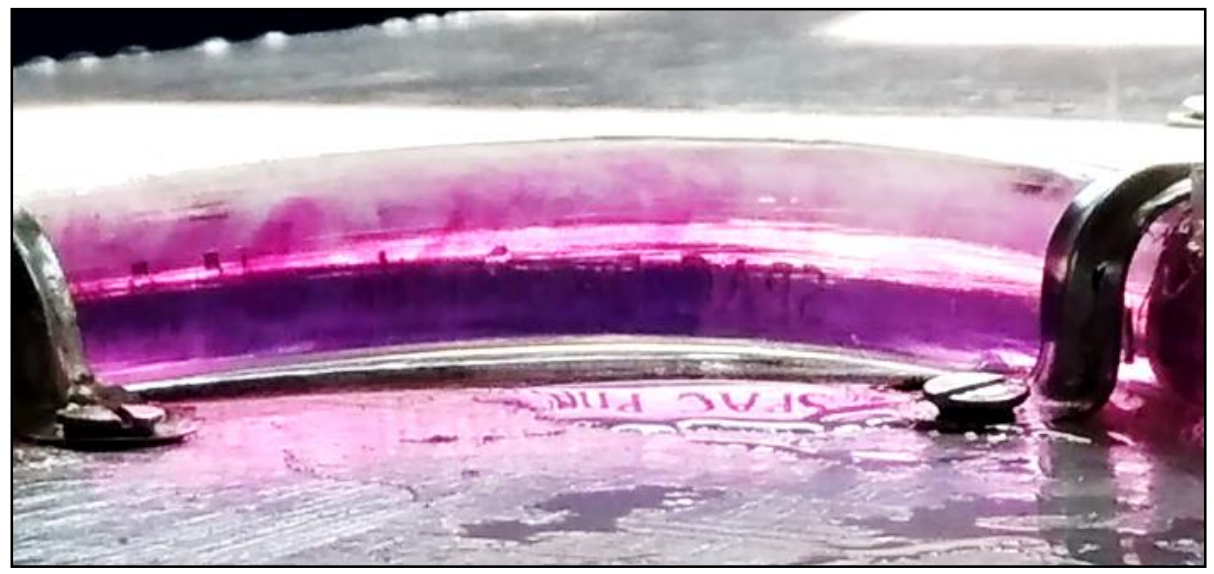

Figure 11. Flow visualization in a diamond base (the portion of the tube between D1 and D2 bend) of Diamond shape tube section

In all test setups, the integral length between two end pressure taps has been maintained to $2.85 \mathrm{~m}$ within subjected to constraints of molding the tube in the desired configurations. The presence of different tube 
configurations has shown their influence on numerical values of pressure drops. The enhanced friction factor has a non-linear relationship with $\mathrm{Re}$ in the laminar regime when plotted for the total length of the tube as shown in figure 12.

Moreover, it can be inferred from the plot that as Re approaches to its threshold of the laminar regime, the steep rise has been experienced in friction factor enhancement. Tube sections with sharp curves and corners have exhibited more pressure drop as expected. Despite the scatter in data in their segment wise plots, the data obtained for total pressure drop measurements have shown good alignment. This could be credited to the compensating effect in developing zones in the entrance tube as well as straight segments in the exit section.

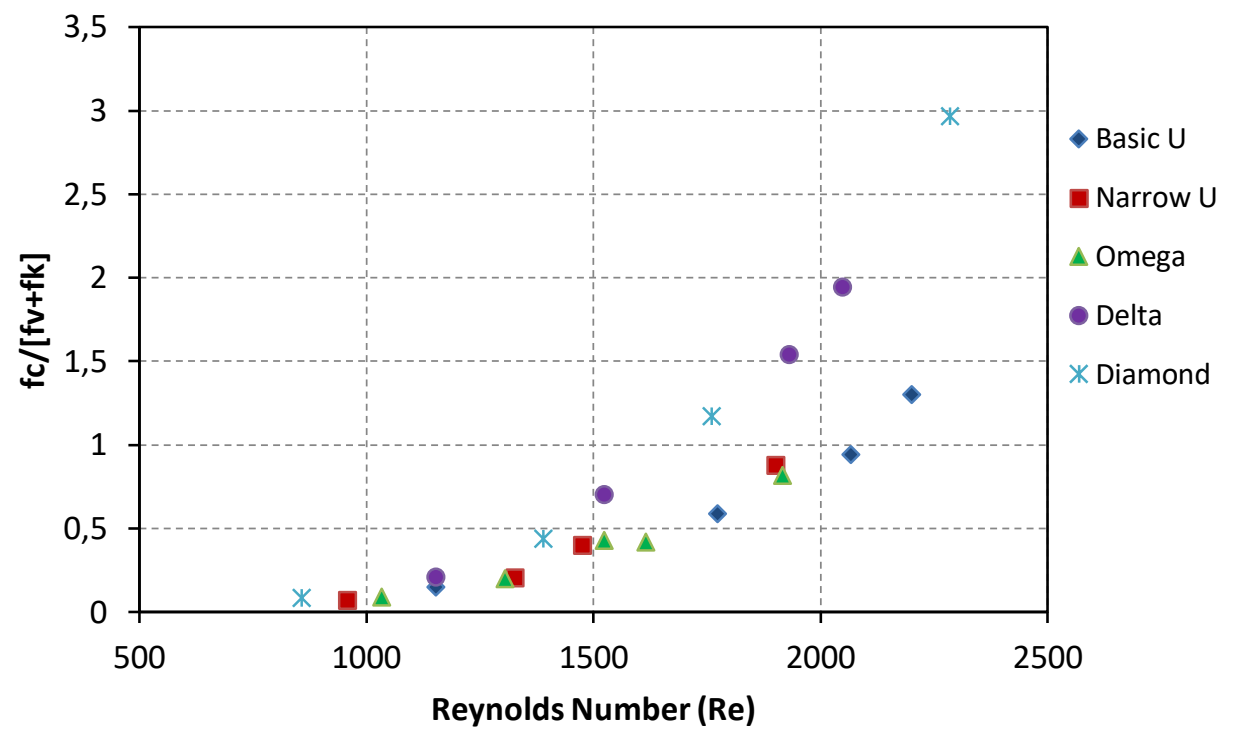

Figure 12. Comparison of friction factor for total length of the tube for various configurations

\section{CONCLUSION}

The test facility was developed for pressure drop characterization of CMFM tube using $\mathrm{CCl}_{4}$ as a manometric fluid. The flow visualization reveals that the flow was laminar, however, local flow transition was observed in the curved sections of the tube. Use of PU material for tube has served the purpose of focusing on pressure drop due to curved sections rather than due to wall friction. Apart from this, the poor thermal conductivity of tube material has helped to ensure quite stable thermo-physical properties like density, viscosity etc. The findings of all experiments can be summarized as below.

1. The response of friction factor in different curved tube sections over the same straight tube has shown similar trends with fundamental distinction in their slopes and then in their numerical values.

2. The tube configurations containing sharper curves and bends has offered the highest enhancements in friction factor throughout the range of Re.

3. Amongst the family of tube section configured with gradual tube curves, steepness in friction factor ratio curve for Omega shape could be due to the absence of a straight segment than observed in its counterparts. It also exhibits the lowest range of pressure drop showing suitability for the measurements in the laminar flow regime.

4. The deviations in low Re, especially Re less than 1000 has been observed owing to small deflection in manometric fluid levels. The scatter of data points at lower Re can be attributed to the transition flow regime induced in the curved sections.

5. For the same length of the test section with sharp curves, the presence of more bends shows more pressure drops. This fact is exhibited in increased pressure drop in case of Diamond shaped test section than in case of Delta shaped test section. The physical phenomenon underlying this is the suffering of flow through transition phase five times (five bends) in Diamond shape than four times (four bends) in Delta shape. 
Journal of Thermal Engineering, Technical Note, Vol. 6, No. 4, pp. 544-558, July, 2020

The trends of friction factors versus Re in the laminar flow regime for five tube sections under study are in stationary conditions of the tube. The flow visualization study in support of these trends can be a precursor to investigate the cause of secondary flow generation in a vibrating tube as reported in the literature. Thus the findings can be a step for resolving the phenomenon of under prediction of mass flow rate in CMFM when used in laminar flow regime through the appropriate selection of tube shape.

\section{ACKNOWLEDGEMENT}

The authors acknowledge the financial support received from Savitribai Phule Pune University, Pune, Maharashtra, India for the conduction of research and technical support by Dr. Satyajit V. Kasar (Indian Institute of Technology, Bombay).

\section{NOMENCLATURE}

$d \quad$ Tube diameter, $\mathrm{m}$

fc Friction factor in the curved segment of the tube

$f k \quad$ Component of friction factor due to change in kinetic energy

$f_{s} \quad$ Friction factor in straight tube

$f_{v} \quad$ Component of friction factor due to viscosity

$l \quad$ Length of tube section, $\mathrm{m}$

$L \quad$ Total Length of the tube, $\mathrm{m}$

$L_{H} \quad$ Hydrodynamic length, $\mathrm{m}$

$n \quad$ Constant in the power law

$r \quad$ Distance from the centerline of the tube towards the wall, $\mathrm{m}$

Re Reynolds number $=\rho \mathrm{Ud} / \mu$

$r_{o} \quad$ Radius of a tube, $\mathrm{m}$

$U \quad$ Average fluid velocity, $\mathrm{m} / \mathrm{s}$

$X \quad$ Distance from inlet bend to outlet bend of tube section, $\mathrm{m}$

$Z \quad$ Dimensionless number $=\mathrm{L} / \mathrm{r}_{\mathrm{o}}$

$\triangle P \quad$ Pressure drop, $\mathrm{Pa}$

$\mu \quad$ Dynamic viscosity of fluid, Pa.s

$\xi \quad$ Dimensionless number $=\mathrm{Z} / \mathrm{Re}$

$\rho \quad$ Density of water, $\mathrm{kg} / \mathrm{m}^{3}$

$\tau \quad$ Shear stress, $\mathrm{N} / \mathrm{mm}^{2}$

\section{REFERENCES}

[1] Kumar V, Anklin M. Numerical simulations of Coriolis flow meters for low Reynolds number flows, J. Metrology Society of India 2011; Vol. 26, no. 3, pp. 225-235.

[2] Bobovnik G, Kutin J, Bajsic I. Estimation of Velocity Profile Effects in the Shell-type Coriolis Flowmeter using CFD Simulations, Journal of Flow Measurement and Instrumentation 2005; Vol. 16, pp. 365-373.

[3] Kutin J, Bobovnik G, Hemp J, Bajsic I. Velocity profile effects in Coriolis mass flow meters: recent findings and open questions, J. Flow Measurement and Instrumentation 2006; Vol. 17, pp. 349-358.

[4] Keulegan G, Beij K. Pressure losses for fluid flow in curved pipes, Part of Journal of research of the National Bureau of Standards 1937; Vol. 18, pp. 89-114.

[5] AL-Nassri S, Unny T. Developing laminar flow in the inlet length of a smooth pipe, J. Applied Scientific Research 1981; Vol. 36, pp. 313-332.

[6] Cuming H. The Secondary Flow in Curved Pipes, Technical report-2880 $(13,979)$ 1955; Aeronautical Research Council, London.

[7] Fargie D, Martin B. Developing Laminar Flow in a Pipe of Circular Cross-section, Proc. Roy. Soc. Lond. A. 1971; 321, pp. 461-476. 
Journal of Thermal Engineering, Technical Note, Vol. 6, No. 4, pp. 544-558, July, 2020

[8] Gupta P, Srinivasan K, Prabhu S. Tests on various configurations of Coriolis mass flow meters, J. Measurement 2006; Vol. 39, pp. 296-307.

[9] Sharma S, Patil P, Vasudev A, Jain S. Performance evaluation of an indigenously designed copper (U) tube Coriolis mass flow sensors, J. Measurement 2010; Vol. 43, pp. 1165-1172.

[10] Ahn H, Uslu I. Experimental investigation on pressure drop in corrugated pipes, Proc. of the ASME 2013, International Mechanical Engineering Congress and Exposition IMECE-2013, San Diego, California, USA.

[11] White FM. Fluid Mechanics, Mc Graw Hill Education 2013; pp. 357-619. 